\title{
Faculty Feedback Analysis System with Efficient Graph-Based Reports
}

\author{
Mohammed Munazzaruddin, Dhandaria Neelam, Mohammed Mateen, Rizwan Khan
}

\begin{abstract}
Feedback plays a vital role in the uprising of any educational institute. With the help of the proper Feedback analysis system the growth rate of the Institute will display an Inclination in its performance. For reputed Institutions which have large number of students, it is not easy to manage a proper feedback system manually, hence there is need for an automation in Faculty Feedback System Faculty Feedback Analysis System is a dynamic web application which is aimed to be anonymous while taking feedback from students and generate efficient and effective reports as prescribed by the NBA \& UGC. The registered students of that particular institute can give the feedback of their faculty of present semester. While giving feedback, the student can see only his/her respective subjects and subject handling faculty names. The feedback form also shows Open Elective and Professional Elective subjects of the students based on their chosen choice. This system also provides the facility to the faculty to check their feedback at any point of time after the feedback is taken. It makes it easier for admin to view reports of all the previously taken feedback. This application is designed in such a way that it can be deployed in real time on a cloud server and can be accessed through smart phones and any small computing devices. All the passwords are encrypted with custom made encryption algorithm to secure the system from all the possible corners. The application has a ready and not ready state which will allow or not allow students to give feedback and will be managed from the admin panel which is beautifully created to be as user-friendly as it can get.The feedback form is also designed in two different models one for large screens where photos of the faculties are also presented with names and another for Smart phones which has its own beautiful custom made design-build for it. Overall performance wise it has been improved over the development cycle starting from version 1.0 to version 4.0.Various types of reports can be generated from the given feedback based on the admin requirements.
\end{abstract}

Keywords - Faculty Feedback Analysis System, feedback Mechanism, graph based reports for feedback, Automatic Feedback system, and anonymous feedback system.

\section{INTRODUCTION}

Faculty Feedback Analysis System was developed in order to overcome the flaws and problems faced in the existing system and most importantly it was developed to reduce the manual work and calculations as much as possible. This system organizes the data inside the database by itself by reading the content from the excel sheets. It calculates the result of the feedback given to each faculty by itself and generates reports in the most efficient way possible.

Revised Manuscript Received on March 5, 2020.

* Correspondence Author

Mohammed Munazzaruddin- Department of CSE, VJIT Hyderabad

DhandariaNeelam Singh Department of CSE, VJIT Hyderabad

Mohammed MateenUddin- Department of CSE, VJIT Hyderabad

Rizwan Khan- Department of CSE, VJIT Hyderabad
We observed many difficulties and flaws in the currently existed mechanism in many aspects. There was no mechanism to allocate open elective and professional elective subjects to students for conducting feedback. It did not have an automated subject faculty assignment mechanism and had to be done Entered manually every semester. The user interface was not user-friendly, with lots of select boxes made it look cluttered on the screen. All the data needed to take feedback had to be entered manually which would consume a lot of time an effort of the faculty. There was no HOD module, Sub-Admin module, individual Faculty Modules. It became much difficulty to individual faculty to check their feedback of the current year as well as of all the previous years. The NBA Expert team was also not satisfied with the reports generated by this system.

In our Proposed System will meet all the requirements proposed by the NBA in terms of report generation. Reports will be generated student wise, subject wise, department wise, year wise and semester wise.

In Addition to this our system is totally anonymous for the admin, which will allow students to give more genuine feedback. Keeping the hard efforts and time in mind the system is developed to be mostly automated which updates all the data just by uploading excel sheets on the application. It will have a fresh look with a highly interactive and beautiful interface which makes it extremely user-friendly for the students. The database is secured with the next level of protection provided to it with all the names given to tables and columns are unique and coded.

The students are being given a proper login and allowed only once to give feedback. This ensures fair feedback to the concerned faculty involved. Feedback timing can also be restricted in order to avoid any kind of misuse either by faculty of students. The feedback system has logging which will keep the record for any kind of misuse of the application with the IP address mapping of the logged in a machine. The system has its own "phpmyadmin" which allows the user to manage the entire database from the application and there is no need for any credentials to the database server of the web application. The system makes use of first ever single insert, update, delete, view module for all the tables. It provides with a checklist to be printed before starting every semester to avoid any repetition of data by uploading file twice. Faculty has its own module where they can $\log$ in and view their feedback at any time after the feedback for that semester is taken. They can view final values for all the subjects for all the semesters combined on the same page any time. HOD has its module integrated into the faculty module and can
Blue Eyes Intelligence Engineering \& Sciences Publication 
view their data and entire faculty of their branch only in terms of points and graph.

Module Description:

The faculty feedback system consists of 5 modules. Each one of them has their own functionalities and their own restrictions when it comes to accessing the application.

The five modules are as follows:

\section{ADMIN \\ SUB-ADMIN \\ HOD \\ FACULTY \\ STUDENT}

All of the modules get their actions logged and can be viewed by admin and sub-admin but none of them can update or delete the log data.

\section{Admin Module:}

Admin is the one who can view all the reports of the faculty taken, this is the higher authority given for the application user. Admin can access almost all of the application except the student module to give feedback.

Feedback data can be viewed for all the semesters previously taken. There is an option called view reports from where you can choose from which year/semester you would like to view the Feedback report. Once you select the year/semester you will be viewing data from the year/semester until you change that again.

\section{Sub-Admin Module:}

Sub-Admin is the one who manages the applications by updating, adding or deleting data required for application to work except feedback data collected from students.

Feedback data is kept secured such that it can only be viewed by Admin, HOD or Faculty and cannot be modified or deleted.

The Sub-Admin module has several functionalities which can be performed only by the sub-admin. All the functions are made clear enough to reduce the work of sub- admin.

\section{HOD Module:}

The module for HOD is mostly similar to faculty module where HOD can view all the reports for the feedback given to him and it also displays reports for all the faculty of the branch to which he/she is HOD.

The reports can be displayed in terms of points and graphs, to access reports in terms of points choose option HOD and to access branch reports in terms of graph choose branch analysis.

HOD can $\log$ in from anywhere and at any time to view department reports or own reports.

\section{Faculty Module:}

Once the feedback for the semester is taken then all the faculty can log in to their respective account and check their feedback report in different types of formats.

It is very much important to have a faculty module where faculty can view their feedback report as the feedback taken is about faculty and they should have the authority to view their feedback at any point of time from anywhere by even using their mobile phones they can view the reports.

Student Module:

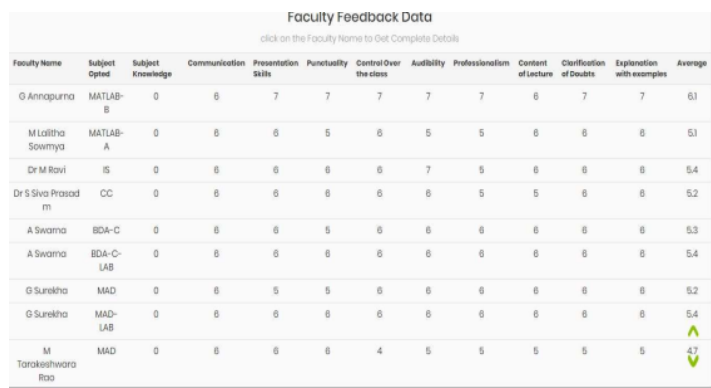

For students to give feedback students module is available from which students can express their views about the faculties by being anonymous.

Student module involves the student to verify their identity whether they are from the same college or not and then followed by setting their passwords and then they got to read some instructions and can start giving feedback.

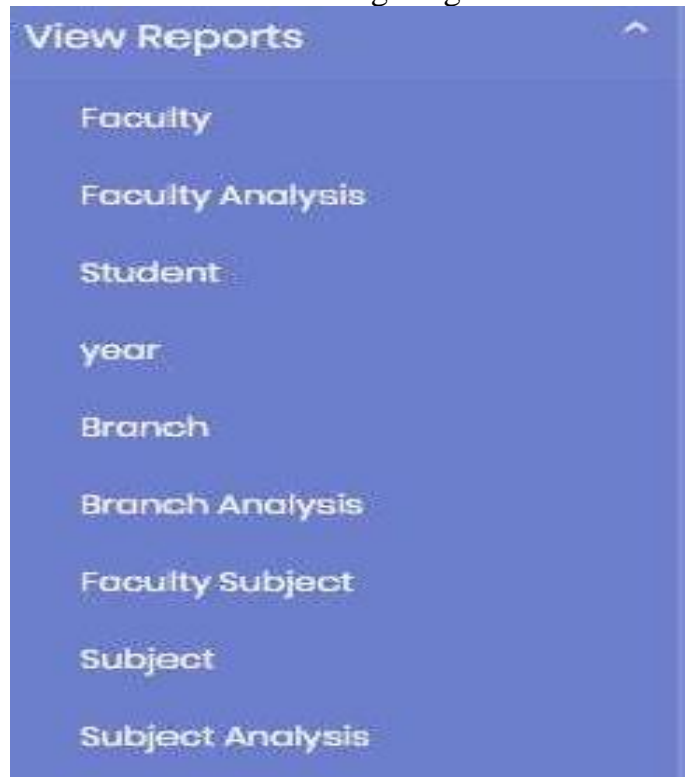

Students can give feedback only once and cannot even login after they have given their feedback. Students have to give feedback to all the faculty for 10 attributes from 1 to 10 . Even if the student logs in by any means his second feedback will not be considered and will be discarded once he submits his inputs.

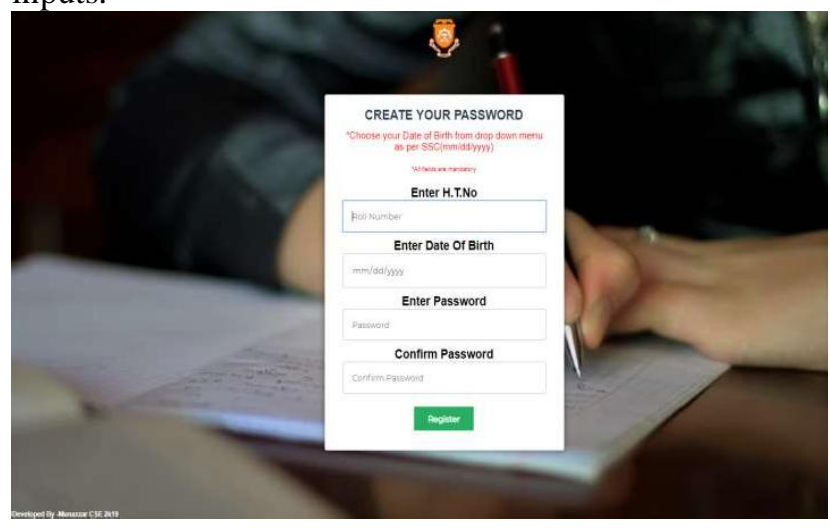




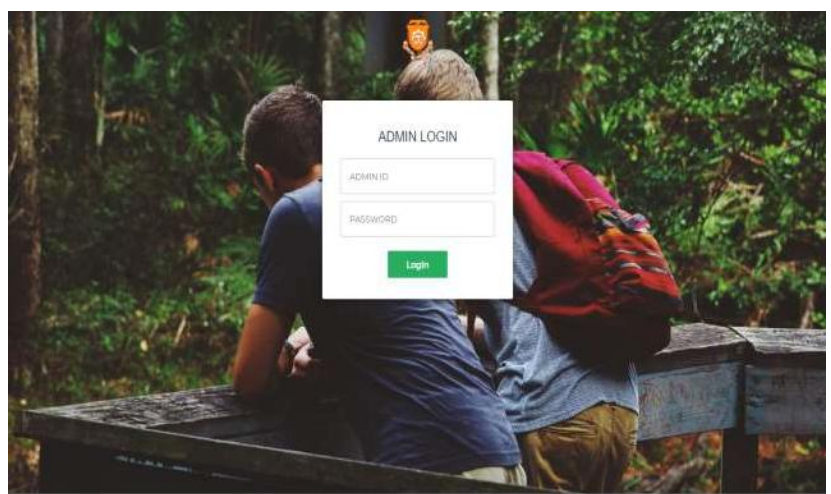

용.

FACULTY FEEDBACK FOR 2018-2019,SemII

You are anonymous to us, Please Feel Free To Give Your Feedback

10-Excellent 9-ExtremelyGood 8-VeryGood 7-Good 6-ModeratelyGood
5-Maderate 4-Tolerable 3-Poor 2-VeryPoor 1-ExtremelyPoor
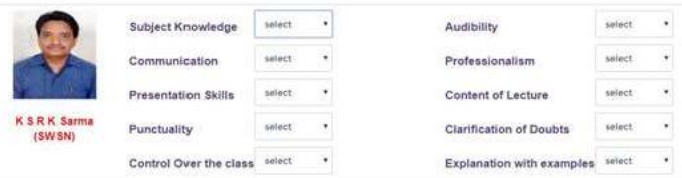

Conment. max 100 characiens
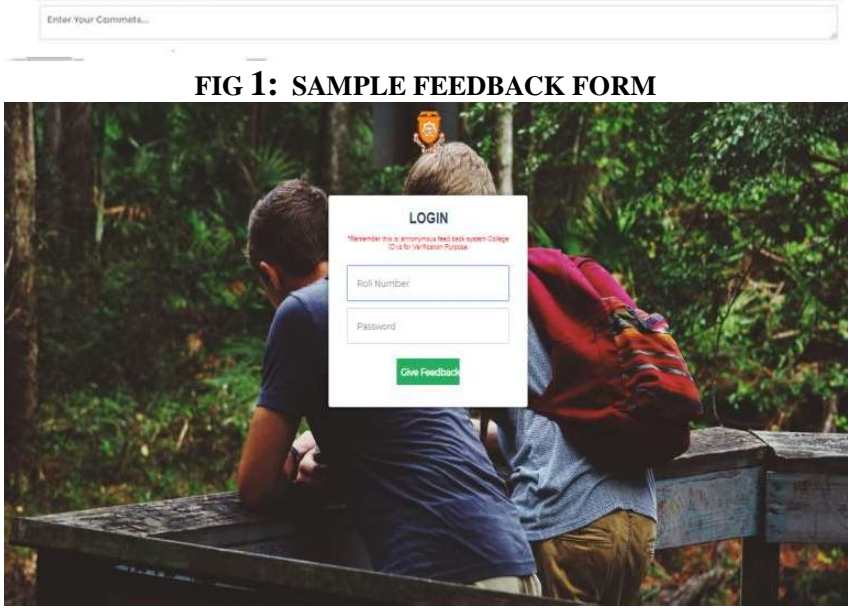

FIG 2: LOGIN FORM

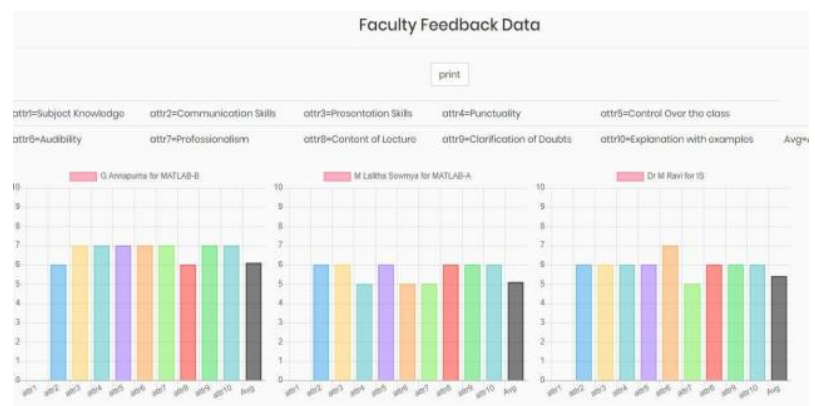

Fig 3: Faculty FEEDBACK DATA

\section{REFERENCES}

1. https://en.wikipedia.org/wiki/Web_ developn

2. https://www.w3schools.com/php/

3. https://www.w3schools.com/js/

4. https://www.w3schools.com/JSON/

5. https://www.w3schools.com/jQuery/

6. https://www.w3schools.com/css/
7. https://github.com/PHPOffice/PHP Excel

8. https:/getbootstrap.com/docs/4.3/ge tting-stad/introduction/

9. http://softwaretestingfundamentals. com/software-testing-methods/

10. http://vjitfeedback.com/

11. https://www.chartjs.org/docs/latest/

\section{AUTHORS PROFILE}

Mr. Mohammed Munazzaruddin completedB.Tech in CSE from Vidya Jyothi Institute of Technology (autonomous) affiliated to JNTUH

Ms. Dhandaria Neelam Singh completed B.Tech in CSE from Vidya jyothi Institute of Technology (autonomous) affiliatedto JNTUH

Mr. Mohammed Mateen Uddin completed B.Tech in CSE from Vidya Jyothi Institute of Technology (autonomous) affiliated to JNTUH

Mr. Rizwan Khan completed B.Tech in CSE from Vidya Jyothi Institute of Technology (autonomous) affiliated to JNTUH

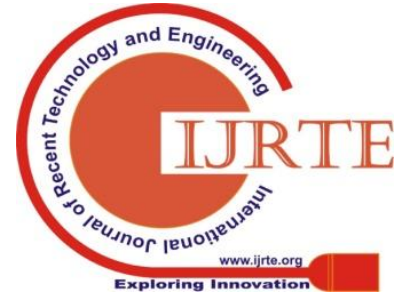

